\title{
Determining Nitrogen Fertilizer Cost Using Turfgrass Response
}

\author{
Travis Wayne Shaddox ${ }^{1}$ and Joseph Bryan Unruh $^{2}$
}

\begin{abstract}
ADDITIONAL INDEX WORDs. bermudagrass, quality, longevity
SuMMARY. Numerous nitrogen $(\mathrm{N})$ sources are used in turfgrass management and vary from soluble to slow-release. Determining the least expensive $\mathrm{N}$ source can be confusing for consumers. Price per ton and price per pound $\mathrm{N}$ are common price comparison methods. An improved approach could use longevity of the $\mathbf{N}$ source to balance the price. The objective of this study was to determine the longevity of turfgrass response to $\mathrm{N}$ sources and to determine the cost to achieve such responses. This study was conducted in Ft. Lauderdale and Jay, FL, from 1 Jan. to 31 Dec. 2018 on 'Riley's Super Sport' (Celebration ${ }^{\circledR}$ ) bermudagrass (Cynodon dactylon). Treatments included nontreated turfgrass, urea, ammonium sulfate, stabilized urea, methylene urea, ureaformaldehyde, two natural organics, sulfur-coated urea, and two polymer-coated urea fertilizers. Treatments were arranged in a split-plot design with $\mathrm{N}$ sources as whole plots and $\mathrm{N}$ rate $(\mathrm{N}$ applied at 49 and $98 \mathrm{~kg} \cdot \mathrm{ha}^{-1}$ every 4 months) as subplots. Turf quality was recorded on a scale of 1 to 9 , where $1=$ dead/brown turf and quality, $6=$ minimal acceptable, and 9 = optimal healthy/green turf. Turf quality ratings were recorded weekly and used to determine response longevity (days quality $\geq 6.0$ ) and area under the turfgrass response curve (AUTRC). Urea resulted in response longevity greater than or equal to other $\mathrm{N}$ sources during each season except when applied at $98 \mathrm{~kg} \cdot \mathrm{ha}^{-1}$ of $\mathrm{N}$ during the fall fertilizer cycle in Jay. Natural organics were $\approx 6$-fold more expensive than urea in Jay and Ft.

Lauderdale using turfgrass response longevity and AUTRC. Urea and sulfurcoated urea were the least expensive soluble and slow-release $\mathrm{N}$ source, respectively, using dollars per pound $\mathrm{N}$, dollars per acre per day, and dollars per acre per quality-day during each fertilizer cycle and annual average in Jay and Ft. Lauderdale. No evidence was found supporting the use of turfgrass response as a more effective method of determining fertilizer cost than dollars per pound $\mathrm{N}$.
\end{abstract}

$\mathrm{T}$ urfgrass often requires supplemental nitrogen $(\mathrm{N})$ applications to maintain acceptable aesthetics and functionality. The average 18-hole golf course in the United States applies $\mathrm{N}$ at $100 \mathrm{~kg} \cdot \mathrm{ha}^{-1}$ per year (Environmental Institute for Golf, 2016). The $\mathrm{N}$ is required to increase production of amino acids and proteins, which leads to increased density and color as well as turfgrass recovery from traffic, drought, and other stresses. Most $\mathrm{N}$ sources used

Received for publication 3 Mar. 2021. Accepted for publication 14 Apr. 2021.

Published online 8 June 2021

${ }^{1}$ Department of Plant and Soil Sciences, University of Kentucky, 417 Plant and Soil Science Building, 1405 Veterans Drive, Lexington, KY 40546

${ }^{2}$ West Florida Research and Education Center, University of Florida, 4235 Experiment Drive, Jay, FL 32565

T.W.S. is the corresponding author. E-mail: shaddox@uky.edu

This is an open access article distributed under the CC BY-NC-ND license (https://creativecommons. org/licenses/by-nc-nd/4.0/).

https://doi.org/10.21273/HORTTECH04837-21 on turfgrasses are either urea or derived from urea (Association of American Plant Food Control Officials, 2017). Since 1998, the cost of urea has fluctuated between $\$ 70$ and $\$ 845$ per ton (Index Mundi, 2019). These costs increase when urea is manufactured into slow-release $\mathrm{N}$ (SRN) because SRN requires the addition of other components such as sulfur or polymer to impart the slowrelease characteristics. However, if turfgrass response to $\mathrm{SRN}$ increases proportional to the cost, then the additional cost of SRN sources may be justified. Confirmation of this postulate would validate a consumer's purchasing decisions and aid in justifying the use of SRN from an economic perspective.

Nitrogen sources may be broadly classified into four groups: soluble, natural organic, reacted, and coated (Association of American Plant Food Control Officials, 2017). Soluble N sources are the least expensive granular $\mathrm{N}$ sources because they do not require the additional manufacturing steps of SRN sources. Natural organics are commonly the least expensive $\mathrm{N}$ source per ton but contain less $\mathrm{N}$ than synthetic $\mathrm{N}$ sources (Carrow et al., 2001). This results in natural organics having the greatest cost per pound $\mathrm{N}$. Examples of reacted $\mathrm{N}$ sources are urea formaldehyde and methylene urea. Because reacted ureas require additional processing, they are more expensive than urea. Coated SRN sources include sulfur-coated urea and polymer-coated urea. Sulfurcoated ureas are normally the least expensive coated $\mathrm{N}$ source because sulfur is less expensive than the polymers necessary to produce polymer-coated ureas (Christians et al., 2017).

It has been well established that $\mathrm{N}$ sources applied correctly consistently increase turfgrass quality. Telenko et al. (2015) applied $\mathrm{N}$ at 49 $\mathrm{kg} \cdot \mathrm{ha}^{-1}$ as urea, polymer-coated urea, and a natural organic on centipedegrass (Eremochloa ophiuroides) in Jay, $\mathrm{FL}$, and reported turfgrass receiving urea resulted in quality greater than or equal to turfgrass receiving polymercoated urea or natural organic during 3 of the 4 years. In addition, Telenko et al. (2015) documented that turf quality of treated plots remained

\begin{tabular}{llll}
\hline $\begin{array}{l}\text { Units } \\
\text { To convert U.S. to SI, } \\
\text { multiply by }\end{array}$ & U.S. unit & SI unit & $\begin{array}{l}\text { To convert SI to U.S., } \\
\text { multiply by }\end{array}$ \\
\hline 0.4047 & acre(s) & $\mathrm{ha}$ & 2.4711 \\
0.3048 & $\mathrm{ft}$ & $\mathrm{m}$ & 3.2808 \\
9.3540 & gal/acre & $\mathrm{L} \cdot \mathrm{ha}^{-1}$ & 0.1069 \\
25.4 & inch $(\mathrm{es})$ & $\mathrm{mm}$ & 0.0394 \\
0.4536 & $\mathrm{lb}$ & $\mathrm{kg}$ & 2.2046 \\
1.1209 & $\mathrm{lb} / \mathrm{acre}$ & $\mathrm{kg} \cdot \mathrm{ha}^{-1}$ & 0.8922 \\
1 & $\mathrm{meq} / 100 \mathrm{~g}$ & $\mathrm{cmol} \cdot \mathrm{kg}^{-1}$ & 1 \\
1 & $\mathrm{mmh} / \mathrm{cm}$ & $\mathrm{mS} \cdot \mathrm{cm}^{-1}$ & 1 \\
1 & $\mathrm{ppm}$ & $\mathrm{mg} \cdot \mathrm{kg}^{-1}$ & 1 \\
0.9072 & ton $(\mathrm{s})$ & $\mathrm{Mg}$ & 1.1023 \\
$\left({ }^{\circ} \mathrm{F}-32\right) \div 1.8$ & ${ }^{\circ} \mathrm{F}$ & ${ }^{\circ} \mathrm{C}$ & $\left({ }^{\circ} \mathrm{C} \times 1.8\right)+32$
\end{tabular}


acceptable during all years regardless of $\mathrm{N}$ source, which implies the cost associated with SRN sources was unnecessary. Shaddox et al. (2016) used centipedegrass to assess the influence of four rates of urea on turf quality and reported that $\mathrm{N}$ applied at $18 \mathrm{~kg} \cdot \mathrm{ha}^{-1}$ per year as urea resulted in acceptable turf quality during each fertilizer cycle over 2 years. Young et al. (1999) reported that $\mathrm{N}$ applied as urea at $49 \mathrm{~kg} \cdot \mathrm{ha}^{-1}$ every $60 \mathrm{~d}$ resulted in st. augustinegrass (Stenotaphrum secundatum) quality greater than or equal to $\mathrm{N}$ applied as polymer-coated urea or biosolid at $98 \mathrm{~kg} \cdot \mathrm{ha}^{-1}$ every $120 \mathrm{~d}$ or at $147 \mathrm{~kg} \cdot \mathrm{ha}^{-1}$ every $180 \mathrm{~d}$. Similarly, Carrow (1997) reported that sulfurcoated urea, polymer-coated urea, reacted urea, and natural organics resulted in average annual turf quality less than or equal to urea during both years of the study. Reports of urea not resulting in acceptable turf quality are scarce and often conditional of $\mathrm{N}$ rate. Trenholm et al. (2012) reported that urea did not result in acceptable st. augustinegrass quality when applied at an $\mathrm{N}$ rate of $49 \mathrm{~kg} \cdot \mathrm{ha}^{-1}$ per year. However, Trenholm et al. (2012) noted that $49 \mathrm{~kg} \cdot \mathrm{ha}^{-1}$ per year was less than the recommended $\mathrm{N}$ rate for st. augustinegrass in central Florida (Trenholm et al., 2011).

Agronomic and environmental benefits of SRN sources have been well established but the cost to acquire these benefits has not been investigated. When properly applied, SRNs may result in reduced $\mathrm{N}$ leaching (Guillard and Kopp, 2004) and reduced applications (Soldat et al., 2008) without reducing turf quality. The use of SRNs has also resulted in as much as $70 \%$ reduction in $\mathrm{N}$ volatilization compared with urea (Del Moro et al., 2017). Advantages of SRN sources have been measured when $\mathrm{N}$ is applied at 98 $\mathrm{kg} \cdot \mathrm{ha}^{-1}$ or greater, which is not commonly recommended using soluble $\mathrm{N}$ sources due to the increased risk of $\mathrm{N}$ loss through runoff or leaching (Shaddox et al., 2016). Thus, in some cases, SRN sources provide additional value relative to urea. However, published studies that have investigated the cost of $\mathrm{N}$ sources applied to warm-season turfgrasses are scant. This is particularly true when the slow-release characteristics and their concomitant influence on turfgrass response are factored in. Determining the cost of $\mathrm{N}$ sources when their slow-release characteristics are taken into account would be useful information for turfgrass managers who desire to save money by applying the least expensive $\mathrm{N}$ source. It has been proposed that the cost "per day of response" based on turfgrass quality should be used to offset the additional cost of SRN. Therefore, the hypothesis of this study was that $\mathrm{N}$ source and rate influence $\mathrm{N}$ fertilizer cost compared with urea when turfgrass response is included in the cost.

\section{Materials and methods}

This study was conducted during 2018 at the University of Florida's Research and Education Centers in Jay, FL (lat. $30^{\circ} 46^{\prime} \mathrm{N}$, long. $87^{\circ} 08^{\prime} \mathrm{W}$ ) and in Ft. Lauderdale, FL (lat. $29^{\circ} 24^{\prime} \mathrm{N}$, long. $\left.82^{\circ} 10^{\prime} \mathrm{W}\right)$. The experimental design was a split plot in a randomized complete block design using four replications. Treatments included nontreated turfgrass and $10 \mathrm{~N}$ sources (Table 1 ), which were applied at $\mathrm{N}$ rates of 49 and $98 \mathrm{~kg} \cdot \mathrm{ha}^{-1}$ every 4 months. Nitrogen sources were assigned to whole plots $(2 \times 3 \mathrm{~m})$ and $\mathrm{N}$ rates were assigned to subplots $(1 \times 3 \mathrm{~m})$. To account for variables not associated with the $\mathrm{N}$ treatments, turf response longevity and AUTRC from nontreated turfgrass were subtracted from treated turfgrass. Treatments were applied on 3 Jan., 4 May, and 3 Sept. in Ft. Lauderdale (year-round growing season) and on 11 Apr. and 1 Aug. in Jay (7-month growing season). Immediately following each treatment application, turfgrass was irrigated with 5 $\mathrm{mm}$ of water. Each treatment application was the beginning of a 4-month fertilizer cycle. Thus, the experiment was repeated three times in Ft. Lauderdale and twice in Jay. Fertilizer cycles were defined as winter fertilizer cycle (WFC), summer fertilizer cycle (SFC), and fall fertilizer cycle (FFC). Treatments were applied by hand to 'Riley's Super Sport' (Celebration ${ }^{\circledR}$ ) bermudagrass (Cynodon dactylon) grown on a Hallandale fine sand (siliceous, hyperthermic Lithic Psammaquent) and on a Fuquay loamy sand (loamy, kaolinitic, thermic Arenic Plinthic Kandiudult) in Ft. Lauderdale and Jay, respectively. The turfgrass in Ft. Lauderdale was established 2 years prior, whereas in Jay the turfgrass had been established for more than 5 years.

Turfgrass was cut at a height of 0.5 inch with a reel mower (9009A TerrainCut Rough Mower; John Deere, Moline, IL) in Ft. Lauderdale and with a reel mower (Toro Reelmaster 3100D;

Table 1. Analysis, derived from source, and average price of nitrogen $(\mathrm{N})$ treatments applied to 'Celebration' bermudagrass in 2018 at Jay and Ft. Lauderdale, FL.

\begin{tabular}{|c|c|c|c|c|c|c|}
\hline \multirow[b]{2}{*}{ N source } & \multirow[b]{2}{*}{$\operatorname{Code}^{\mathrm{z}}$} & \multirow[b]{2}{*}{ Derived from } & \multirow[b]{2}{*}{$\mathbf{N}-\mathbf{P}-\mathrm{K}^{\mathrm{y}}$} & \multirow[b]{2}{*}{ Source } & \multicolumn{2}{|c|}{ Avg price ${ }^{x}$} \\
\hline & & & & & $\$ /$ ton & $\$ / 1 b \mathrm{~N}$ \\
\hline Urea & - & Urea & $46-0-0$ & Yara International, Oslo, Norway & 643 & 0.70 \\
\hline Ammonium sulfate & - & Ammonium sulfate & $21-0-0$ & Yara International & 537 & 1.28 \\
\hline UFlexx & - & Stabilized urea & $46-0-0$ & Koch Agronomic Services, Wichita, KS & 1,140 & 1.27 \\
\hline $\mathrm{XCU}$ & - & Sulfur-coated urea & $43-0-0$ & Koch Agronomic Services & 950 & 1.10 \\
\hline Methylene urea & - & Methylene urea & $40-0-0$ & Koch Agronomic Services & 1,385 & 1.73 \\
\hline Urea formaldehyde & - & Urea formaldehyde & $38-0-0$ & Koch Agronomic Services & 1,376 & 1.81 \\
\hline Milorganite & NATl & Natural organic & $6-0.8-0$ & Milorganite, Milwaukee, WI & 533 & 4.44 \\
\hline Houactinite & NAT2 & Natural organic & $5-1.7-0$ & Harrell's, Lakeland, FL & 334 & 3.34 \\
\hline Duration-90 & PCUl & Polymer-coated urea & $43-0-0$ & Koch Agronomic Services & 1,200 & 1.40 \\
\hline Polyon-42 & PCU2 & Polymer-coated urea & $42-0-0$ & Koch Agronomic Services & 1,430 & 1.70 \\
\hline
\end{tabular}

${ }^{\mathrm{z}} \mathrm{NAT}=$ natural organic, $\mathrm{PCU}=$ polymer-coated urea .

${ }^{\mathrm{y}} \mathrm{N}$-phosphorus-potassium.

${ }^{\mathrm{x}} \$ 1 / \mathrm{ton}=\$ 1.1023 / \mathrm{Mg}, \$ 1 / \mathrm{lb}=\$ 2.2046 / \mathrm{kg}$. 
The Toro Company, Bloomington, $\mathrm{MN}$ ) in Jay. Post and preemergence herbicides, fungicides, and insecticides were applied uniformly to all plots as needed to prevent pest damage. Irrigation supplied water equivalent to $80 \%$ of the previous week's reference evapotranspiration and was automatically stopped for $24 \mathrm{~h}$ when rainfall exceeded $6 \mathrm{~mm}$ during any 24-h period. Following each treatment application, granular potassium sulfate (Harrell's, Lakeland, FL) was applied to all plots using a potassium rate of $49 \mathrm{~kg} \cdot \mathrm{ha}^{-1}$. To balance the phosphorus provided by the natural organic l (NATl) and natural organic 2 (NAT2), granular concentrated superphosphate (Harrell's) was applied at a phosphorus rate of 30 $\mathrm{kg} \cdot \mathrm{ha}^{-1}$ to all plots except plots receiving NAT1 or NAT2. Micronutrients were applied every $90 \mathrm{~d}$ using a micronutrient solution (Max Minors; Harrell's) applied at $14 \mathrm{~L} \cdot \mathrm{ha}^{-1}$.

Turf quality was recorded weekly on a scale of 1 to 9 , where $1=$ dead $/$ brown turf and $9=$ optimal healthy/ green turf. Turf quality ratings $\geq 6$ were considered acceptable (Throssell et al., 2009). Turfgrass response longevity was determined as day's turfgrass quality $\geq 6.0$. The AUTRC was calculated using graphing software (GraphPad Prism; GraphPad Software, San Diego, CA) to provide a measure of both response longevity and magnitude. The following formula (GraphPad Software, 2020) was used to calculate AUTRC:

Ft. Lauderdale

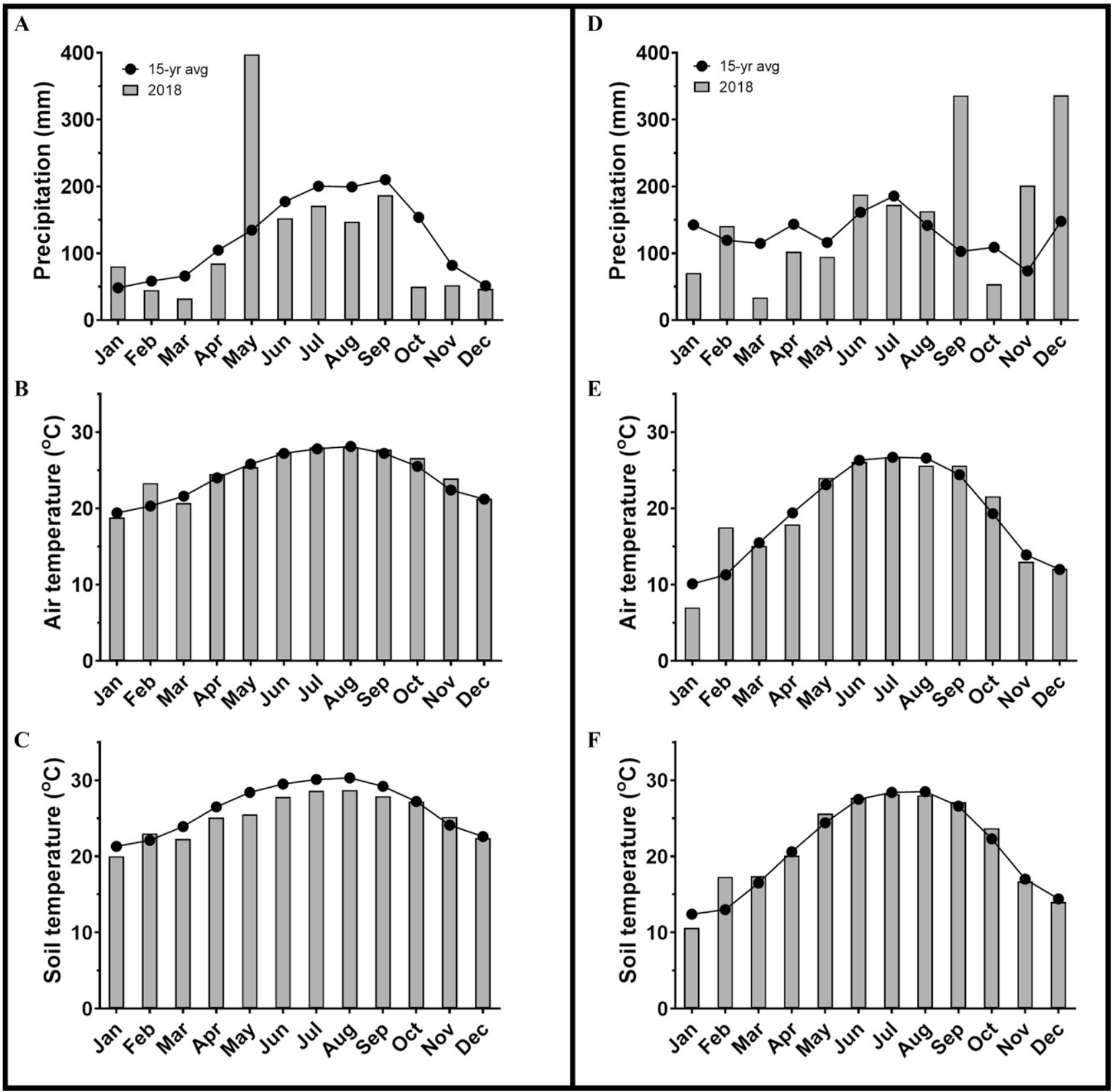

Fig. 1. Ft. Lauderdale, FL, average monthly (A) precipitation, (B) air temperature, and (C) soil temperature; and Jay, FL (D) precipitation, $(\mathrm{E})$ air temperature, and $(\mathrm{F})$ soil temperature in $2018 ;\left(1.8 \times{ }^{\circ} \mathrm{C}\right)+32={ }^{\circ} \mathrm{F}, 1 \mathrm{~mm}=0.0394$ inch. 
Table 2. Chemical properties of Fuquay loamy sand and Hallandale fine sand from Jay and Ft. Lauderdale, FL, respectively.

\begin{tabular}{|c|c|c|}
\hline Parameter & Fuquay loamy sand & Hallandale fine sand \\
\hline \multicolumn{3}{|c|}{$\overline{\text { Mehlich III content }\left(\mathrm{mg} \cdot \mathrm{kg}^{-1}\right)^{\mathrm{z}}}$} \\
\hline Phosphorus & 33.0 & 20.0 \\
\hline Potassium & 38.0 & 14.0 \\
\hline Calcium & 481.0 & 1495.0 \\
\hline Magnesium & 81.0 & 24.0 \\
\hline Sodium & 7.0 & 8.0 \\
\hline Zinc & 4.4 & 1.5 \\
\hline Manganese & 30.0 & 4.0 \\
\hline $\operatorname{CEC~}\left(\mathrm{cmol} \cdot \mathrm{kg}^{-1}\right)^{\mathrm{y}}$ & 4.6 & 7.7 \\
\hline $\mathrm{pH}_{\mathrm{KCl}}{ }^{\mathrm{x}}$ & 5.3 & 7.0 \\
\hline $\mathrm{EC}\left(\mathrm{mS} \cdot \mathrm{cm}^{-1}\right)^{\mathrm{w}}$ & 0.1 & 0.1 \\
\hline $\mathrm{OM}(\%)^{\mathrm{v}}$ & 3.4 & 2.1 \\
\hline
\end{tabular}

${ }^{\mathrm{Z}}$ Nutrients extracted using the Mehlich III extractant (Mehlich, 1984); $1 \mathrm{mg} \cdot \mathrm{kg}^{-1}=1 \mathrm{ppm}$.

${ }^{\mathrm{y}}$ Cation exchange capacity by ammonium acetate $\mathrm{pH} 7.0 ; 1 \mathrm{cmol} \cdot \mathrm{kg}^{-1}=1 \mathrm{meq} / 100 \mathrm{~g}$.

${ }^{x} 1: 1$ (soil:1 M potassium chloride).

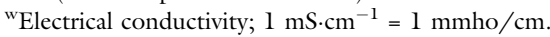

${ }^{v}$ Organic matter by weight loss on ignition.

$$
\Sigma^{\mathrm{ni}-1}{ }_{\mathrm{i}=1}\left\{\left[\mathrm{y}_{i}+\mathrm{y}_{(i+1)}\right] / 2\right\} \cdot\left(\mathrm{t}_{(i+1)}-\mathrm{t}_{i}\right)
$$

where $i$ is the order index at the $i$ th observation, $\mathrm{y}_{i}$ is the turf quality rating, $\mathrm{t}_{i}$ is the time in days at the $i$ th observation, and $n$ is the number of observations. The AUTRC when turf quality was $<6$ was excluded from the total AUTRC calculation and the baseline was set at 5. Thus, GraphPad Prism recognized turf qualities of $6,7,8$, and 9 as units $1,2,3$, and 4 , respectively. To this end, the maximum annual AUTRC was 1460 quality-days, which was equivalent to a turf quality of 9 for $365 \mathrm{~d}(4 \times 365)$.

Fertilizer sale prices were provided by four Florida fertilizer distributors except for polymer-coated urea 1 (PCU1) and polymer-coated urea 2 (PCU2) because only one distributor of PCUl provided a sale price and PCU2 was a proprietary product sold by only one distributor. The average sale price was used for cost analysis. Fertilizer cost per day of response (dollars per acre per day) and cost per AUTRC (dollars per acre per quality-day) were determined by dividing the $\mathrm{N}$ source cost (dollars per pound $\mathrm{N}$ ) by the response days or AUTRC, respectively.

Weather data were collected during the duration of the research from an on-site weather network system (Institute of Food and Agricultural Science, 2018), which provided meteorological information in 15-min intervals. Data collection stations were located within $100 \mathrm{~m}$ of the study locations.

Model residuals were analyzed for normality both numerically and healthy/green turf.

${ }^{\mathrm{y}} \$ 1 /$ acre $=\$ 2.4711 /$ ha. to analyze data, and mean separations were conducted using the TukeyKramer procedure for multiple comparisons $(P \leq 0.05)$. Linear regressions of fertilizer cost, response longevity, and AUTRC were conducted on raw data using GraphPad Prism.

\section{Results and discussion}

Air and soil temperatures were similar to the 15-year monthly averages as shown in Fig. 1. Precipitation was similar to the 15-year average with three exceptions. During May in Ft. Lauderdale and during September and December in Jay, precipitation exceeded the 15-year average by $\approx 3$ fold. As shown in Table 2, the Fuquay soil chemical properties indicated the Mehlich III content was greater than or equal to the minimum suggested thresholds for phosphorus and potassium for turfgrass (Liu et al., 2008; Sartain, 2002), whereas the Hallandale soil contained sufficient levels of Mehlich III phosphorus but contained less than the minimum recommended potassium concentration.

Table 3. Type III tests for fixed effects of turfgrass response longevity, area under the turfgrass response curve (AUTRC), and cost when applied to 'Celebration' bermudagrass in 2018 at Jay and Ft. Lauderdale, FL, during winter, summer, and fall fertilizer cycles (WFC, SFC, and FFC, respectively).

\begin{tabular}{|c|c|c|c|c|c|c|c|c|c|}
\hline & df & WFC & SFC & FFC & Annual & WFC & SFC & FFC & Annual \\
\hline & \multicolumn{5}{|c|}{ Duration of turf quality $\geq 6.0^{\mathrm{z}}$} & \multicolumn{4}{|c|}{ AUTRC } \\
\hline & \multicolumn{5}{|c|}{ d } & \multicolumn{4}{|c|}{ quality-days } \\
\hline \multicolumn{10}{|l|}{ Jay } \\
\hline $\begin{array}{l}\text { Nitrogen } \\
\quad \text { source }(\mathrm{NS})\end{array}$ & 9 & - & NS & NS & NS & - & $* * *$ & $* *$ & ** \\
\hline $\begin{array}{l}\text { Nitrogen } \\
\quad \text { rate }(\mathrm{NR})\end{array}$ & 1 & - & $*$ & NS & $*$ & - & $* *$ & $* *$ & ** \\
\hline $\mathrm{NS} \times \mathrm{NR}$ & 9 & - & NS & $*$ & NS & - & NS & $*$ & NS \\
\hline \multicolumn{10}{|l|}{ Ft. Lauderdale } \\
\hline NS & 9 & NS & $*$ & NS & NS & $*$ & $* * *$ & NS & $* * *$ \\
\hline NR & 1 & NS & NS & NS & NS & NS & $*$ & NS & NS \\
\hline \multirow[t]{3}{*}{$\mathrm{NS} \times \mathrm{NR}$} & 9 & NS & NS & NS & NS & NS & NS & NS & NS \\
\hline & \multicolumn{9}{|c|}{ Fertilizer $\cos \mathrm{t}^{\mathrm{y}}$} \\
\hline & & & acre 1 & day & & $\$ /$ & cre pe & $r$ qualit & ty-day \\
\hline
\end{tabular}

\begin{tabular}{llllllllll}
\hline Jay & & & & & & & & & \\
NS & 9 & - & NS & NS & $* * *$ & - & NS & $* * *$ & $* * *$ \\
NR & 1 & - & NS & NS & NS & - & NS & $*$ & NS \\
NS $\times$ NR & 9 & - & NS & NS & NS & - & NS & NS & NS \\
Ft. Lauderdale & & & & & & & & & \\
NS & 9 & NS & $* * *$ & $* * *$ & $* * *$ & NS & $* * *$ & NS & $* * *$ \\
NR & 1 & NS & NS & NS & NS & NS & NS & NS & NS \\
NS $\times$ NR & 9 & NS & NS & NS & NS & NS & NS & NS & NS \\
\hline
\end{tabular}

${ }^{\mathrm{z}}$ Turf quality on a scale of $\mathrm{l}$ to 9 , where $\mathrm{l}=$ dead/brown turf, $6=$ minimal acceptable, and $9=$ optimal

Ns, *,*****Nonsignificant or significant at $P \leq 0.05,0.01$, or 0.001 , respectively. 
Table 4. Days of turfgrass response and area under the turfgrass response curve (AUTRC) as influenced by nitrogen source and rate on 'Celebration' bermudagrass in 2018 at Jay, FL.

\begin{tabular}{|c|c|c|c|c|}
\hline & $\mathrm{SFC}^{\mathrm{z}}$ & Annual $^{\mathrm{y}}$ & SFC & Annual \\
\hline & \multicolumn{2}{|c|}{$\begin{array}{l}\text { Duration of turf } \\
\text { quality } \geq 6.0^{x}\end{array}$} & \multicolumn{2}{|c|}{ AUTRC } \\
\hline & \multicolumn{2}{|c|}{ d } & \multicolumn{2}{|c|}{ quality-days } \\
\hline \multicolumn{5}{|l|}{ Nitrogen source ${ }^{\mathrm{w}}$} \\
\hline Urea & $40 \mathrm{a}^{\mathrm{v}}$ & $122 \mathrm{a}$ & $53 \mathrm{~b}$ & $202 \mathrm{~b}$ \\
\hline Ammonium sulfate & $53 \mathrm{a}$ & 138 a & $69 \mathrm{~b}$ & $225 \mathrm{ab}$ \\
\hline Stabilized urea & $45 \mathrm{a}$ & $122 \mathrm{a}$ & $66 \mathrm{~b}$ & $208 \mathrm{~b}$ \\
\hline Sulfur-coated urea & $52 \mathrm{a}$ & $140 \mathrm{a}$ & $77 \mathrm{ab}$ & $243 \mathrm{ab}$ \\
\hline Methylene urea & $52 \mathrm{a}$ & $146 \mathrm{a}$ & $75 \mathrm{ab}$ & $253 \mathrm{ab}$ \\
\hline Urea formaldehyde & $53 \mathrm{a}$ & $143 \mathrm{a}$ & $89 \mathrm{ab}$ & $265 \mathrm{ab}$ \\
\hline Natural organic 1 & $53 \mathrm{a}$ & $142 \mathrm{a}$ & $69 \mathrm{~b}$ & $236 \mathrm{ab}$ \\
\hline Natural organic 2 & $43 \mathrm{a}$ & $133 \mathrm{a}$ & $61 \mathrm{~b}$ & $226 \mathrm{ab}$ \\
\hline Polymer-coated urea 1 & $64 \mathrm{a}$ & 152 a & $117 \mathrm{a}$ & $279 \mathrm{a}$ \\
\hline Polymer-coated urea 2 & $61 \mathrm{a}$ & 141 a & $117 \mathrm{a}$ & $260 \mathrm{ab}$ \\
\hline \multicolumn{5}{|l|}{ Nitrogen rate $\left(\mathrm{kg} \cdot \mathrm{ha}^{-1}\right)^{\mathrm{u}}$} \\
\hline 49 & $42 \mathrm{~b}$ & $123 \mathrm{~b}$ & $57 \mathrm{~b}$ & $187 \mathrm{~b}$ \\
\hline 98 & $61 \mathrm{a}$ & $153 \mathrm{a}$ & $101 \mathrm{a}$ & 293 a \\
\hline \multicolumn{5}{|c|}{$\begin{array}{l}{ }^{ } \mathrm{SFC}=\text { summer fertilizer cycle. } \\
\text { yCumulative. } \\
{ }^{\mathrm{x}} \text { Turf quality on a scale of } 1 \text { to } 9 \text {, where } 1=\text { dead/brown turf, } 6=\text { minimal acceptable, and } 9=\text { optimal } \\
\text { healthy/green turf. } \\
\text { wAmmonium sulfate (Yara International, Oslo, Norway), stabilized urea (UFlexx; Koch Agronomic Services, } \\
\text { Wichita, KS), sulfur-coated urea (XCU; Koch Agronomic Services), methylene urea (Koch Agronomic Serv- } \\
\text { ices), urea formaldehyde (Koch Agronomic Services), natural organic } 1 \text { (Milorganite, Milwaukee, WI), natural } \\
\text { organic } 2 \text { (Houactinite; Harrell's, Lakeland, FL), polymer-coated urea } 1 \text { (Duration-90; Koch Agronomic Serv- } \\
\text { ices), polymer-coated urea } 2 \text { (Polyon- } 42 \text {, Koch Agronomic Services). } \\
\text { "Within a column, means followed by a common letter are not significantly different according to the Tukey- } \\
\text { Kramer test at the } 5 \% \text { significance level. } \\
\text { "1 } 1 \mathrm{~kg} \cdot \mathrm{ha}^{-1}=0.8922 \mathrm{lb} / \text { acre. }\end{array}$} \\
\hline
\end{tabular}

Turfgrass response longevity and AUTRC were not influenced by the $\mathrm{N}$ source $\times \mathrm{N}$ rate interaction except during the FFC in Jay (Table 3 ). Therefore, the influence of $\mathrm{N}$ source and $\mathrm{N}$ rate are presented by main effects except during FFC in Jay in which the influence of $\mathrm{N}$ source is presented within $\mathrm{N}$ rates. Fertilizer cost was not influenced by the $\mathrm{N}$ source $\times \mathrm{N}$ rate interaction and, thus, results are reported by main effects.

\section{Turf response longevity}

The annual cumulative response longevity was not influenced by $\mathrm{N}$ source in Jay or Ft. Lauderdale (Tables 4 and 5 ) and ranged from 122 to $152 \mathrm{~d}$ and 191 to $245 \mathrm{~d}$, respectively. In addition, response longevity was similar among $\mathrm{N}$ sources during the SFC in Jay and during the WFC and FFC in Ft. Lauderdale. However, during the SFC in Ft. Lauderdale, urea and stabilized urea resulted in a 1.5 -fold increase in response longevity compared with methylene urea. In Jay during the FFC, NAT2 resulted in a 1.3 -fold increase in response longevity compared with urea and ammonium sulfate when $\mathrm{N}$ was applied at $98 \mathrm{~kg} \cdot \mathrm{ha}^{-1}$ (Table 6). Nitrogen applied at $98 \mathrm{~kg} \cdot \mathrm{ha}^{-1}$ resulted in a 1.4-fold and a 1.2-fold increase in

Table 5. Duration of turfgrass response and area under the turfgrass response curve (AUTRC) as influenced by nitrogen source and rate on 'Celebration' bermudagrass in 2018 at Ft. Lauderdale, FL.

\begin{tabular}{|c|c|c|c|c|c|c|c|c|}
\hline & $\mathrm{WFC}^{\mathbf{z}}$ & $\mathrm{SFC}^{\mathrm{z}}$ & $\mathrm{FFC}^{\mathbf{z}}$ & Annual $^{\mathrm{y}}$ & WFC & SFC & FFC & Annual \\
\hline & \multicolumn{4}{|c|}{ Duration of turf quality $\geq 6.0^{x}$} & \multicolumn{4}{|c|}{ AUTRC } \\
\hline & \multicolumn{4}{|c|}{ d } & \multicolumn{4}{|c|}{ quality-days } \\
\hline \multicolumn{9}{|l|}{ Nitrogen source ${ }^{w}$} \\
\hline Urea & $56 \mathrm{a}^{\mathrm{v}}$ & 99 a & $73 \mathrm{a}$ & $230 \mathrm{a}$ & $104 \mathrm{abc}$ & 198 a & $114 \mathrm{a}$ & $416 \mathrm{ab}$ \\
\hline Ammonium sulfate & $65 \mathrm{a}$ & $90 \mathrm{ab}$ & $76 \mathrm{a}$ & $231 \mathrm{a}$ & $134 \mathrm{a}$ & $191 \mathrm{a}$ & $130 \mathrm{a}$ & $456 \mathrm{a}$ \\
\hline Methylene urea & $43 a$ & $67 \mathrm{~b}$ & $80 \mathrm{a}$ & $191 \mathrm{a}$ & $67 \mathrm{c}$ & $104 \mathrm{~d}$ & $130 \mathrm{a}$ & $302 \mathrm{c}$ \\
\hline Urea formaldehyde & $46 a$ & $84 \mathrm{ab}$ & $83 a$ & $214 \mathrm{a}$ & 72 bc & $125 \mathrm{dc}$ & $124 \mathrm{a}$ & $322 \mathrm{bc}$ \\
\hline Natural organic 1 & $55 \mathrm{a}$ & $80 \mathrm{ab}$ & $74 \mathrm{a}$ & $210 \mathrm{a}$ & $101 \mathrm{abc}$ & $135 \mathrm{bcd}$ & $126 \mathrm{a}$ & $363 \mathrm{abc}$ \\
\hline Natural organic 2 & $64 a$ & $93 \mathrm{ab}$ & $87 \mathrm{a}$ & $245 \mathrm{a}$ & $129 \mathrm{ab}$ & $144 \mathrm{a}-\mathrm{d}$ & $127 \mathrm{a}$ & $401 \mathrm{ab}$ \\
\hline Polymer-coated urea 1 & $52 \mathrm{a}$ & $71 \mathrm{ab}$ & $86 a$ & 209 a & $103 \mathrm{abc}$ & $102 \mathrm{~d}$ & $134 \mathrm{a}$ & $340 \mathrm{bc}$ \\
\hline Polymer-coated urea 2 & $66 \mathrm{a}$ & $77 \mathrm{ab}$ & $73 \mathrm{a}$ & $217 \mathrm{a}$ & $115 \mathrm{abc}$ & $116 \mathrm{~cd}$ & $114 \mathrm{a}$ & $345 \mathrm{bc}$ \\
\hline \multicolumn{9}{|l|}{ Nitrogen rate $\left(\mathrm{kg} \cdot \mathrm{ha}^{-1}\right)^{\mathrm{u}}$} \\
\hline
\end{tabular}

${ }^{\mathrm{z}} \mathrm{WFC}=$ winter fertilizer, $\mathrm{SFC}=$ spring fertilizer cycle, $\mathrm{FFC}=$ summer fertilizer cycle.

y Cumulative.

${ }^{\mathrm{x}}$ Turf quality on a scale of 1 to 9 , where $1=$ dead/brown turf and quality, $6=$ minimal acceptable, and $9=$ optimal healthy $/$ green turf.

${ }^{w}$ Ammonium sulfate (Yara International, Oslo, Norway), stabilized urea (UFlexx; Koch Agronomic Services, Wichita, KS), sulfur-coated urea (XCU; Koch Agronomic Services), methylene urea (Koch Agronomic Services), urea formaldehyde (Koch Agronomic Services), natural organic 1 (Milorganite, Milwaukee, WI), natural organic 2 (Houactinite; Harrell's, Lakeland, FL), polymer-coated urea 1 (Duration-90; Koch Agronomic Services), polymer-coated urea 2 (Polyon-42; Koch Agronomic Services).

${ }^{v}$ Within a column, means followed by a common letter are not significantly different according to the Tukey-Kramer test at the $5 \%$ significance level.

${ }^{\mathrm{u}} \mathrm{l} \mathrm{kg} \cdot \mathrm{ha}^{-1}=0.8922 \mathrm{lb} / \mathrm{acre}$. 
Table 6. Days of turfgrass response and area under the turfgrass response curve (AUTRC) as influenced by the interaction of nitrogen source and rate on 'Celebration' bermudagrass in 2018 at Jay, FL, during the fall fertilizer cycle.

\begin{tabular}{|c|c|c|c|c|}
\hline & \multicolumn{4}{|c|}{ Nitrogen rate $\left(\mathrm{kg} \cdot \mathrm{ha}^{-1}\right)^{\mathrm{z}}$} \\
\hline & 49 & 98 & 49 & 98 \\
\hline & \multicolumn{2}{|c|}{$\begin{array}{l}\text { Duration of turf } \\
\text { quality } \geq 6.0^{y}\end{array}$} & \multicolumn{2}{|c|}{ AUTRC } \\
\hline & \multicolumn{2}{|c|}{$\mathrm{d}$} & \multicolumn{2}{|c|}{ quality-days } \\
\hline \multicolumn{5}{|l|}{ Nitrogen source $\mathrm{e}^{\mathrm{x}}$} \\
\hline Urea & $85 \mathrm{a}^{\mathrm{w}}$ & $79 \mathrm{~b}$ & $131 \mathrm{a}$ & $167 \mathrm{c}$ \\
\hline Ammonium sulfate & 89 a & $79 \mathrm{~b}$ & $135 \mathrm{a}$ & $177 \mathrm{bc}$ \\
\hline Stabilized urea & $70 \mathrm{a}$ & $84 \mathrm{ab}$ & $109 \mathrm{a}$ & $175 \mathrm{bc}$ \\
\hline Sulfur-coated urea & $78 \mathrm{a}$ & $97 \mathrm{ab}$ & $145 \mathrm{a}$ & $187 \mathrm{abc}$ \\
\hline Methylene urea & $87 \mathrm{a}$ & $100 \mathrm{ab}$ & $140 \mathrm{a}$ & $214 \mathrm{ab}$ \\
\hline Urea formaldehyde & $79 \mathrm{a}$ & $101 \mathrm{ab}$ & $126 \mathrm{a}$ & $224 \mathrm{a}$ \\
\hline Natural organic 1 & $83 \mathrm{a}$ & $95 \mathrm{ab}$ & $131 \mathrm{a}$ & $202 \mathrm{abc}$ \\
\hline Natural organic 2 & $73 \mathrm{a}$ & $106 \mathrm{a}$ & $115 \mathrm{a}$ & $213 \mathrm{ab}$ \\
\hline Polymer-coated urea 1 & $87 \mathrm{a}$ & $89 \mathrm{ab}$ & $139 a$ & $185 \mathrm{abc}$ \\
\hline Polymer-coated urea 2 & $74 \mathrm{a}$ & $85 \mathrm{ab}$ & $122 \mathrm{a}$ & $163 c$ \\
\hline
\end{tabular}

${ }^{\mathrm{z}} \mathrm{l} \mathrm{kg} \cdot \mathrm{ha}^{-1}=0.8922 \mathrm{lb} / \mathrm{acre}$.

${ }^{\mathrm{y}}$ Turf quality on a scale of 1 to 9 , where $1=$ dead/brown turf, $6=$ minimal acceptable, and $9=$ optimal healthy/green turf.

${ }^{\mathrm{x}}$ Ammonium sulfate (Yara International, Oslo, Norway), stabilized urea (UFlexx; Koch Agronomic Services, Wichita, KS), sulfur-coated urea (XCU; Koch Agronomic Services), methylene urea (Koch Agronomic Services), urea formaldehyde (Koch Agronomic Services), natural organic l (Milorganite, Milwaukee, WI), natural organic 2 (Houactinite; Harrell's, Lakeland, FL), polymer-coated urea 1 (Duration-90; Koch Agronomic Services), polymer-coated urea 2 (Polyon-42; Koch Agronomic Services).

wWithin a column, means followed by a common letter are not significantly different according to the TukeyKramer test at the $5 \%$ significance level.

response longevity compared with $\mathrm{N}$ applied at $49 \mathrm{~kg} \cdot \mathrm{ha}^{-1}$ in Jay during the SFC and annual cumulative (Table 4), whereas $\mathrm{N}$ rate did not influence response longevity in Ft. Lauderdale (Table 5).

Determining turfgrass response longevity by measuring the days turf quality $\geq 6$ has not been previously reported. Other researchers have documented the influence of $\mathrm{N}$ sources on turf quality over time, but these studies were not designed to determine the number of response days (Carrow, 1997; Cisar et al., 2001). However, when previous research reports turf quality by months over 1 year, response longevity can be inferred. These results compare favorably with those reported by Soldat et al. (2008), who documented turfgrass quality in response to slow and soluble N sources on kentucky bluegrass (Poa pratensis), a blend of creeping bentgrass (Agrostis stolonifera) with some annual bluegrass (Poa annua), and a blend of kentucky bluegrass and fine fescue (Festuca sp.) in New York and reported that stabilized urea (UFlexx; Koch Turf \& Ornamental, Wichita, KS), methylene urea, and urea formaldehyde resulted in similar response longevity (days turf quality $\geq 6$ ) as urea on each sward. Differing results were reported by Young et al. (1999) who measured the influence of $\mathrm{N}$ sources on st. augustinegrass in Ft. Lauderdale. Young et al. (1999) reported that between April and November, $\mathrm{N}$ applied at $49 \mathrm{~kg} \cdot \mathrm{ha}^{-1}$ every 2 months or $98 \mathrm{~kg} \cdot \mathrm{ha}^{-1}$ every 4 months applied as polymer-coated urea resulted in $32 \mathrm{~d}$ fewer compared with urea. The reduction in response days occurred during the $32 \mathrm{~d}$ following the initial treatment applications. In the current study, an initial delayed turfgrass response resulting from polymercoated ureas was not observed and, thus, $\mathrm{N}$ sources resulted in similar response longevities.

The AUTRC was influenced by $\mathrm{N}$ sources during most cycles and the annual cumulative in Jay and Ft. Lauderdale (Tables 4 and 5). In Jay during the SFC, the polymer-coated ureas resulted in a 2.2 -fold increase in AUTRC compared with urea. In addition, PCUl resulted in a 1.4 -fold increase in the annual cumulative AUTRC compared with urea. In Ft. Lauderdale, urea resulted in AUTRC greater than or equal to other $\mathrm{N}$ sources during each fertilizer cycle and the annual cumulative. Furthermore in Ft. Lauderdale, ammonium sulfate resulted in a $40 \%$ increase in AUTRC compared with reacted ureas. Because ammonium sulfate did not result in increased response longevity compared with reacted ureas, the increased AUTRC was a result of ammonium sulfate increasing turf quality. Reacted ureas contain water-insoluble $\mathrm{N}$, which requires microbial degradation to become plant available. The microbial degradation process has been identified as a causal factor in reducing turfgrass quality, color, and $\mathrm{N}$ uptake from reacted ureas when compared with urea, especially during the initial weeks following application (Landschoot and Waddington, 1987). However, when applied for multiple years, reacted ureas have produced turf quality greater than or equal to urea (Carrow, 1997). Presumably, continued applications of reacted ureas allows the water-insoluble $\mathrm{N}$ from prior years to degrade and increase plant available $\mathrm{N}$. This accumulation effect has not been documented using AUTRC. Single-year studies, such as the current study, are not capable of capturing this potential benefit of SRNs and, thus, multiyear studies that measure turfgrass response to $\mathrm{N}$ fertilizers using AUTRC would be valuable. Differences among $\mathrm{N}$ sources were documented using annual cumulative AUTRC, whereas differences among $\mathrm{N}$ sources were not documented using annual cumulative days of response in either Jay or Ft. Lauderdale. This indicates that using AUTRC may be more useful than response longevity when comparing the agronomic value of $\mathrm{N}$ sources because AUTRC accounts for both longevity and magnitude of response.

The cost of urea was less than or equal to other $\mathrm{N}$ sources during each fertilizer cycle and annual cumulative in Jay and Ft. Lauderdale using dollars per acre per day and dollars per acre per quality-day (Tables 7 and 8). Among slow-release $\mathrm{N}$ sources, the cost of sulfur-coated urea was less than or equal to other slow-release $\mathrm{N}$ sources during each fertilizer cycle and when averaged annually. In Jay, $\mathrm{N}$ sources resulted in similar dollars per acre per day during the SFC and FFC; however, natural organics resulted in a 4 -fold increase in the annual average dollars per acre per day 
Table 7 . Fertilizer cost as influenced by nitrogen source and rate on 'Celebration' bermudagrass in 2018 at Jay, FL.

\begin{tabular}{|c|c|c|c|c|c|c|}
\hline & $\mathrm{SFC}^{\mathrm{z}}$ & $\mathrm{FFC}^{\mathbf{z}}$ & Annual $^{\mathrm{y}}$ & SFC & FFC & Annual $^{\mathrm{x}}$ \\
\hline & \multicolumn{3}{|c|}{$\$ /$ acre per day ${ }^{v}$} & \multicolumn{3}{|c|}{$\$ /$ acre per quality-day } \\
\hline \multicolumn{7}{|l|}{ Nitrogen source ${ }^{w}$} \\
\hline Ammonium sulfate & $1.69 \mathrm{a}$ & $1.08 \mathrm{a}$ & $1.25 \mathrm{~b}$ & $1.36 \mathrm{a}$ & 0.54 cde & $0.74 \mathrm{~b}$ \\
\hline Stabilized urea & $8.62 \mathrm{a}$ & $1.19 \mathrm{a}$ & $1.62 \mathrm{~b}$ & $2.86 \mathrm{a}$ & 0.59 cde & $0.84 \mathrm{~b}$ \\
\hline Sulfur-coated urea & $4.20 \mathrm{a}$ & $0.84 \mathrm{a}$ & $1.13 \mathrm{~b}$ & $6.83 \mathrm{a}$ & $0.42 \mathrm{de}$ & $0.60 \mathrm{~b}$ \\
\hline Natural organic 1 & $13.72 \mathrm{a}$ & $3.61 \mathrm{a}$ & $5.03 \mathrm{a}$ & $11.91 \mathrm{a}$ & $1.82 \mathrm{a}$ & $2.76 \mathrm{a}$ \\
\hline Natural organic 2 & $17.34 \mathrm{a}$ & $2.75 \mathrm{a}$ & $4.16 \mathrm{a}$ & $10.12 \mathrm{a}$ & $1.42 \mathrm{~b}$ & $2.29 \mathrm{a}$ \\
\hline Polymer-coated urea 1 & $1.72 \mathrm{a}$ & $1.20 \mathrm{a}$ & $1.39 \mathrm{~b}$ & $0.88 \mathrm{a}$ & $0.58 \mathrm{cde}$ & $0.68 \mathrm{~b}$ \\
\hline Polymer-coated urea 2 & $2.15 \mathrm{a}$ & $1.75 \mathrm{a}$ & $1.89 \mathrm{~b}$ & $1.03 \mathrm{a}$ & $0.81 \mathrm{c}$ & $0.89 \mathrm{~b}$ \\
\hline \multicolumn{7}{|l|}{ Nitrogen rate $\left(\mathrm{kg} \cdot \mathrm{ha}^{-1}\right)^{\mathrm{t}}$} \\
\hline
\end{tabular}

${ }^{\mathrm{z}} \mathrm{SFC}=$ summer fertilizer cycle, $\mathrm{FFC}=$ fall fertilizer cycle.

${ }^{\mathrm{y}}$ (Total annual days turfgrass quality $\left.\geq 6.0\right) /$ (total annual nitrogen cost).

(Total annual area under the turfgrass response curve) / (total annual nitrogen cost).

${ }^{w}$ Ammonium sulfate (Yara International, Oslo, Norway), stabilized urea (UFlexx; Koch Agronomic Services, Wichita, KS), sulfur-coated urea (XCU; Koch Agronomic Services), methylene urea (Koch Agronomic Services), urea formaldehyde (Koch Agronomic Services), natural organic 1 (Milorganite, Milwaukee, WI), natural organic 2 (Houactinite; Harrell's, Lakeland, FL), polymer-coated urea 1 (Duration-90; Koch Agronomic Services), polymer-coated urea 2 (Polyon-42; Koch Agronomic Services). ${ }^{\mathrm{v}} \$ 1 / \mathrm{acre}=\$ 2.4711 / \mathrm{ha}$.

${ }^{\mathrm{u}}$ Within a column, means followed by a common letter are not significantly different according to the Tukey-Kramer test at the $5 \%$ significance level.

${ }^{\mathrm{t}} \mathrm{l} \mathrm{kg} \cdot \mathrm{ha}^{-1}=0.8922 \mathrm{lb} /$ acre.

Table 8. Fertilizer cost as influenced by nitrogen source and rate on 'Celebration' bermudagrass in 2018 at Ft. Lauderdale, FL.

\begin{tabular}{|c|c|c|c|c|c|c|c|c|}
\hline & $\mathrm{WFC}^{\mathrm{z}}$ & $\mathrm{SFC}^{\mathrm{z}}$ & $\mathrm{FFC}^{\mathrm{z}}$ & Annual $^{\mathrm{y}}$ & WFC & SFC & FFC & Annual $^{\mathrm{x}}$ \\
\hline & \multicolumn{4}{|c|}{$\$ /$ acre per day ${ }^{v}$} & \multicolumn{4}{|c|}{$\$$ /acre per quality-day } \\
\hline \multicolumn{9}{|l|}{ Nitrogen source $^{w}$} \\
\hline Ammonium sulfate & $1.50 \mathrm{a}$ & $0.92 \mathrm{bc}$ & $1.20 \mathrm{~b}$ & $1.09 \mathrm{~cd}$ & $0.63 \mathrm{a}$ & $0.43 \mathrm{bc}$ & $0.67 \mathrm{a}$ & $0.54 \mathrm{~cd}$ \\
\hline Stabilized urea & $1.37 \mathrm{a}$ & $0.85 \mathrm{bc}$ & $1.36 \mathrm{~b}$ & $1.09 \mathrm{~cd}$ & $0.78 \mathrm{a}$ & $0.50 \mathrm{bc}$ & $0.81 \mathrm{a}$ & $0.63 \mathrm{~cd}$ \\
\hline Sulfur-coated urea & $4.18 \mathrm{a}$ & $0.78 \mathrm{bc}$ & $1.01 \mathrm{~b}$ & $1.00 \mathrm{~cd}$ & $2.67 \mathrm{a}$ & $0.43 \mathrm{bc}$ & $0.67 \mathrm{a}$ & $0.58 \mathrm{~cd}$ \\
\hline Natural organic 1 & $10.01 \mathrm{a}$ & $4.73 \mathrm{a}$ & $5.37 \mathrm{a}$ & $4.69 \mathrm{a}$ & $4.85 \mathrm{a}$ & $3.05 \mathrm{a}$ & $9.90 \mathrm{a}$ & $2.70 \mathrm{a}$ \\
\hline Natural organic 2 & $9.36 \mathrm{a}$ & $3.14 \mathrm{ab}$ & $2.73 \mathrm{ab}$ & $3.09 \mathrm{~b}$ & $5.10 \mathrm{a}$ & $2.18 \mathrm{ab}$ & $1.81 \mathrm{a}$ & $1.90 \mathrm{~b}$ \\
\hline Polymer-coated urea 1 & $2.12 \mathrm{a}$ & $1.94 \mathrm{bc}$ & $1.17 \mathrm{~b}$ & $1.34 \mathrm{~cd}$ & $1.08 \mathrm{a}$ & $1.34 \mathrm{abc}$ & $0.72 \mathrm{a}$ & $0.82 \mathrm{~cd}$ \\
\hline Polymer-coated urea 2 & $1.84 \mathrm{a}$ & $1.70 \mathrm{bc}$ & $1.68 \mathrm{~b}$ & $1.58 \mathrm{~cd}$ & $1.11 \mathrm{a}$ & $1.19 \mathrm{abc}$ & $0.97 \mathrm{a}$ & $0.98 \mathrm{~cd}$ \\
\hline \multicolumn{9}{|l|}{ Nitrogen rate $\left(\mathrm{kg} \cdot \mathrm{ha}^{-1}\right)^{\mathrm{t}}$} \\
\hline
\end{tabular}

${ }^{\mathrm{z}} \mathrm{WFC}=$ winter fertilizer cycle, $\mathrm{SFC}=$ summer fertilizer cycle, $\mathrm{FFC}=$ fall fertilizer cycle.

${ }^{\mathrm{y}}$ (Total annual days turfgrass quality $\left.\geq 6.0\right) /$ (total annual nitrogen cost).

(Total annual area under the turfgrass response curve) / (total annual nitrogen cost).

${ }^{w}$ Ammonium sulfate (Yara International, Oslo, Norway), stabilized urea (UFlexx; Koch Agronomic Services, Wichita, KS), sulfur-coated urea (XCU; Koch Agronomic Services), methylene urea (Koch Agronomic Services), urea formaldehyde (Koch Agronomic Services), natural organic 1 (Milorganite, Milwaukee, WI), natural organic 2 (Houactinite; Harrell's, Lakeland, FL), polymer-coated urea 1 (Duration-90; Koch Agronomic Services), polymer-coated urea 2 (Polyon-42; Koch Agronomic Services).

${ }^{\mathrm{v}} \$ 1 / \mathrm{acre}=\$ 2.4711 / \mathrm{ha}$.

${ }^{\text {u} W i t h i n ~ a ~ c o l u m n, ~ m e a n s ~ f o l l o w e d ~ b y ~ a ~ c o m m o n ~ l e t t e r ~ a r e ~ n o t ~ s i g n i f i c a n t l y ~ d i f f e r e n t ~ a c c o r d i n g ~ t o ~ t h e ~ T u k e y-K r a m e r ~ t e s t ~ a t ~ t h e ~} 5 \%$ significance level.

${ }^{\mathrm{t}} \mathrm{l} \mathrm{kg} \cdot \mathrm{ha}^{-1}=0.8922 \mathrm{lb} / \mathrm{acre}$.

compared with urea. In Jay during the FFC, NAT1, NAT2, PCU2, and urea formaldehyde resulted in 5.5-, 4.3-, 2.5-, and 2.1-fold increase in dollars per acre per quality-day compared with urea, respectively. In addition, both natural organics were $\approx 4$.6-fold more expensive than urea when averaged annually. Similar to Jay, natural organics in Ft. Lauderdale were $\approx 6$-fold more expensive than urea when using the annual average cost of either measurement method. Because natural organics resulted in response days and AUTRC similar to urea except for NATl during SFC in Ft. Lauderdale, the increased cost of natural organics was a result of their sale price (dollars per pound $\mathrm{N}$ ), which was also $\approx 6$-fold more expensive than urea. 

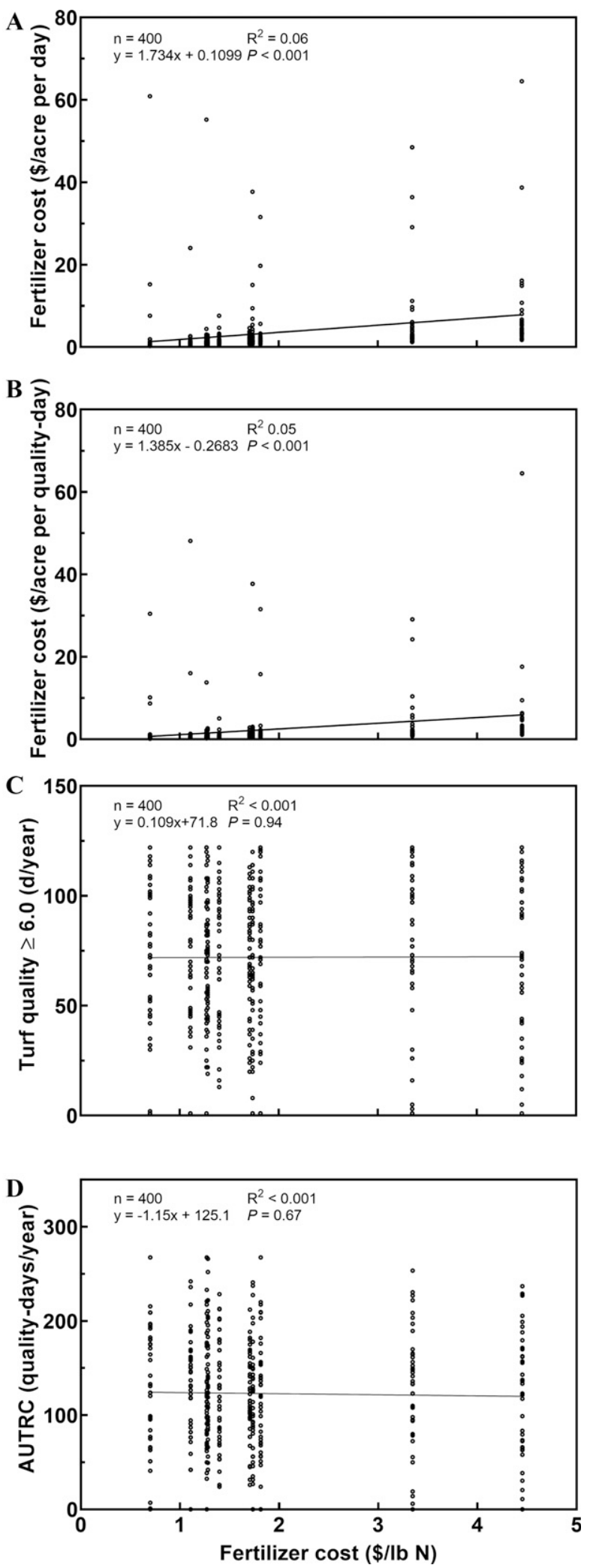

Fig. 2. Relationship between nitrogen $(N)$ fertilizer cost per pound and (A) $N$ fertilizer cost per acre per day, (B) $\mathrm{N}$ fertilizer cost per acre per quality-day, (C) turfgrass response longevity, and (D) area under the turfgrass response curve (AUTRC) in 2018 at Jay and Ft. Lauderdale, FL. Regression was conducted on raw data. Turf quality on a scale of 1 to 9 , where $1=$ dead/brown turf and quality, $6=$ minimal acceptable, and $9=$ optimal healthy $/$ green turf; $\$ 1 / 1 \mathrm{~b}=$ $\$ 2.2046 / \mathrm{kg}, \$ 1 /$ acre $=\$ 2.4711 /$ ha.
Regression of dollars per pound $\mathrm{N}$ with dollars per acre per day and dollars per acre per quality-day resulted in significant positive yet weak relationships (Fig. $2 \mathrm{~A}$ and $\mathrm{B}$ ). This indicates that using the initial fertilizer cost in dollars per pound $\mathrm{N}$ to accurately predict the fertilizer cost in dollars per acre per day and dollars per acre per quality-day would be unlikely using the regression model developed under these experimental conditions. Last, regressing the dollars per pound $\mathrm{N}$ with response longevity (Fig. 2C) and AUTRC (Fig. 2D) determined these variables were unrelated. These results support the proposition that inexpensive $\mathrm{N}$ sources can result in similar response longevity and AUTRC as more expensive $\mathrm{N}$ sources.

\section{Conclusions}

The only $\mathrm{N}$ source that increased turfgrass response longevity compared with urea was NAT2 applied at an N rate of $98 \mathrm{~kg} \cdot \mathrm{ha}^{-1}$ during the FFC in Jay. During all remaining seasons and annual cumulative, $\mathrm{N}$ sources did not increase turfgrass response longevity compared with urea. Differences among $\mathrm{N}$ sources were more frequently measured using AUTRC indicating that using AUTRC may be more useful than response longevity when determining the influence of $\mathrm{N}$ sources on turfgrass. Urea was less than or equal to the cost of other $\mathrm{N}$ sources during each fertilizer cycle and annual average in Jay and Ft. Lauderdale. Regression analysis indicated that fertilizer cost in dollars per acre per day and dollars per acre per quality-day increases as dollars per pound $\mathrm{N}$ increases but turf response longevity and AUTRC may not increase concomitantly. These results indicate that urea and sulfur-coated urea are the least expensive soluble and slow-release $\mathrm{N}$ sources, respectively. Evidence from this study supports the use of dollars per pound $\mathrm{N}$ when comparing $\mathrm{N}$ source costs, whereas this study found no evidence that using turfgrass response provided a more accurate $\mathrm{N}$ source cost comparison than dollars per pound $\mathrm{N}$.

\section{Literature cited}

Association of American Plant Food Control Officials. 2017. Official publication. AAPFCO, West Lafayette, IN. 
Carrow, R.N. 1997. Turfgrass response to slow-release nitrogen fertilizers. Agron. J. 89:491-496, doi: 10.2134/agronj1997.00 021962008900030020x.

Carrow, R.N., D.V. Waddington, and P.E. Rieke. 2001. Turfgrass soil fertility and chemical problems: Assessment and management. Ann Arbor Press, Chelsea, MI.

Christians, N., A.J. Patton, and Q.D. Law. 2017. Fundamentals of turfgrass management. Wiley, Hoboken, NJ.

Cisar, J.L., G.H. Snyder, J.J. Haydu, and K.E. Williams. 2001. Turf response to coated-urea fertilizers. II. Nitrogen content in clippings, nitrogen uptake, and nitrogen retention from prills. Intl. Turfgrass Soc. Res. J. 9:368-374.

Del Moro, S.K., D.M. Sullivan, and D.A. Horneck. 2017. Ammonia volatilization from broadcast urea and alternative dry nitrogen fertilizers. Soil Sci. Soc. Amer. J. 81:1629-1639, doi: 10.2136/sssaj2017. 06.0181 .

Environmental Institute for Golf. 2016. Golf course environmental profile: Nutrient use and management on U.S. golf courses. Golf Course Superintendents Assoc. Amer., Lawerence, KS.

GraphPad Software. 2020. GraphPad Prism 8 user guide. GraphPad Software, San Diego, CA.

Guillard, K. and K.L. Kopp. 2004. Nitrogen fertilizer form and associated nitrate leaching from cool-season lawn turf. J. Environ.
Qual. 33:1822-1827, doi: 10.2134/jeq 2004.1822.

Index Mundi. 2019. Commodity prices: Urea. 7 Aug. 2019. < https://www.index mundi.com $/$ commodities $/$ ?commodity= urea\&months $=360>$.

Institute of Food and Agricultural Science. 2018. Florida automated weather network. 1 Aug. 2020. <http://fawn.ifas.ufl.edu>.

Landschoot, P.J. and D.V. Waddington. 1987. Response of turfgrass to various nitrogen sources. Soil Sci. Soc. Amer. J. 51:225-230, doi: 10.2136/sssaj1987.036 15995005100010046x.

Liu, M., J.B. Sartain, L.E. Trenholm, and G.L. Miller. 2008. Phosphorus requirements of st. augustinegrass grown in sandy soils. Crop Sci. 48:1178-1186, doi: 10.2135/cropsci2007.09.0506.

Mehlich, A. 1984. Mehlich 3 soil test extractant: A modification of Mehlich 2 extractant. Commun. Soil Sci. Plant Anal. 15:1409-1416, doi: 10.1080/00103628 409367568 .

Sartain, J.B. 2002. Tifway bermudagrass response to potassium fertilization. Crop Sci. 42:507-512, doi: $10.2135 /$ cropsci 2002.5070 .

Shaddox, T.W., J.B. Unruh, L.E. Trenholm, P. McGroary, and J.L. Cisar. 2016. Nitrogen rate required for acceptable st. augustinegrass and associated nitrate leaching. Crop Sci. 56:439-451, doi: 10.2135/ cropsci2015.04.0226.
Soldat, D.J., A.M. Petrovic, and J. Barlow. 2008. Turfgrass response to nitrogen sources with varying nitrogen release rates. Acta Hort. 783:453-462, doi: 10.17660/Acta Hortic.2008.783.47.

Telenko, D.E.P., T.W. Shaddox, J.B. Unruh, and L.E. Trenholm. 2015. Nitrate leaching, turf quality, and growth rate of 'Floratam' st. augustinegrass and common centipedegrass. Crop Sci. 55:1320-1328, doi: $10.2135 /$ cropsci2014.09.0639.

Throssell, C.S., G.T. Lyman, M.E. Johnson, G.A. Stacey, and C.D. Brown. 2009. Golf course environmental profile measures nutrient use and management and fertilizer restrictions, storage, and equipment calibration. Appl. Turf. Sci. 6:1-22, doi: 10.1094/ATS-2009-1203-01-RS.

Trenholm, L.E., J.L. Cisar, and J.B. Sartain. 2011. St. augustinegrass for Florida lawns. 7 Aug. 2019. <http://edis.ifas.ufl. edu/lh010 $>$.

Trenholm, L.E., J.B. Unruh, and J.B. Sartain. 2012. Nitrate leaching and turf quality in established 'Floratam' st. augustinegrass and 'Empire' zoysiagrass. J. Environ. Qual. 41:793-799, doi: 10.2134/ jeq2011.0183.

Young, N.G., J.L. Cisar, J.E. Erickson, G.H. Snyder, J.B. Sartain, and K.E. Williams. 1999. St. augustinegrass response to nitrogen sources under contrasting application rates and frequency. Intl. Turfgrass Soc. Res. J. 11:121-136. 